\title{
Auditory Brainstem Response at the Detection Limit
}

\author{
Bernd LÜtkenhÖNer ${ }^{1}$ And AnNemarie Seither-Preisler ${ }^{1,2}$ \\ ${ }^{1}$ Section of Experimental Audiology, ENT Clinic, Münster University Hospital, Kardinal-von-Galen-Ring 10, 48129, \\ Münster, Germany \\ ${ }^{2}$ Department of Psychology, Cognitive Science Section, University of Graz, Graz, Austria
}

Received: 15 December 2006; Accepted: 1 July 2008; Online publication: 14 August 2008

\begin{abstract}
Specific predictions regarding the level dependence of auditory evoked responses near the detection limit were made in a companion modeling study (Lütkenhöner, J Assoc Res Otolaryngol 9:102-121, 2008). Here, these predictions are experimentally tested for auditory brainstem responses (ABR) to Gaussian-shaped 4-kHz tone pulses (full width at half maximum $=0.5 \mathrm{~ms}$ ) that were presented at sound levels close to the subjective threshold. In the average of over about one million stimulus repetitions (repetition period $=16 \mathrm{~ms}$ ), the amplitude of $A B R$ wave $V$ showed a smooth transition from a proportional to a logarithmic growth with increasing sound intensity. The latter type of growth corresponds to a linear increase with respect to sound level measured in decibels. Alternatively, the ABR amplitude near the detection limit may be considered a linear function of sound pressure, although - according to the modelthis is only an approximation. Data and model are consistent with the view that a sensory threshold does not exist for the auditory modality, in accordance with signal detection theory. Even so, the model may be used to define a quasithreshold that is comparable to the subjective threshold.
\end{abstract}

Keywords: hearing threshold, electrophysiological threshold, ABR threshold, brainstem auditory evoked potential, AEP, signal detection theory, linearity in the auditory system

Correspondence to: Bernd Lütkenhöner ${ }^{1}$ ? $\cdot$ Section of Experimental Audiology, ENT Clinic $\cdot$ Münster University Hospital · Kardinal-vonGalen-Ring 10, 48129, Münster, Germany. Telephone: +49-2518356864; fax: +49-251-8356882; email: Lutkenh@uni-muenster.de

\section{INTRODUCTION}

If the frequency of a tone is close to the characteristic frequency $(\mathrm{CF})$ of the cochlear location being studied, an increasing sound level results in a highly compressive growth of basilar membrane velocity. But regardless of $\mathrm{CF}$, basilar membrane velocity is proportional to sound pressure at very low levels (see, e.g., the review by Robles and Ruggero 2001). The firing rate of auditory nerve fibers shows a linear growth at low levels, too, but the independent variable appears to be an integer power of sound pressure. In mammals, the stimulus-driven component of the firing rate seems to be proportional to sound pressure squared, corresponding to sound intensity (Yates 1990; Yates et al. 1990). The same type of growth was found in the DC response of inner hair cells (Dallos 1985). A recent modeling study suggests that the effect is related to the nonlinear gating of the transducer current (Lopez-Poveda and Eustaquio-Martin 2006). A quadratic dependence on sound pressure was also observed in the nonmammallian cochlea, although a minority of fibers showed proportionality to the cube of sound pressure (Eatock et al. 1991).

At very low levels, the compound action potential (CAP) of the auditory nerve can be expected to exhibit basically the same intensity dependence as the driven rates of the most sensitive fibers. Thus, we would expect the CAP to grow, at very low levels, in proportion to intensity. The same may be supposed for auditory evoked potentials (AEP) of more central origin because in the low-intensity limit the discharge probabilities at the various levels of the auditory pathway may be assumed to be linearly related. Published data, however, appear to be inconsistent with this conjecture as they suggest a linear relationship between response amplitude and sound level in decibels (see, e.g., 
Eggermont and Odenthal 1974; Elberling and Don 1987; Versnel et al. 1992; Lütkenhöner and Klein 2007). This means that the response amplitude seems to increase with the logarithm of sound intensity. Such a logarithmic law implies, furthermore, the existence of a threshold because a function linearly increasing with sound level inevitably becomes 0 at a specific decibel value. This corollary is inconsistent with signal detection theory, a theory that denies the existence of a sensory threshold (Swets 1961).

The above contradictions might have a simple explanation. The postulated proportionality between intensity and response amplitude possibly only applies to intensities that are significantly below those considered in previous AEP studies. This hypothesis gave rise to the present study in which we considered the auditory brainstem response (ABR) to a short tone pulse. Measuring at very low intensities implies that the amplitude of the recorded AEP is very small and a reasonable signal-to-noise ratio can only be achieved by a compensatory increase of the number of averages. It soon became clear to us that collecting robust data would require an exorbitant measuring time, rendering the intended experiment almost unfeasible. The problem was overcome in an unconventional way. In the most ambitious experiment, the first author served as the one and only subject while doing desk work (e.g., reviewing manuscripts). Hence, measuring time was not an issue anymore, and by repeating the same experiment again and again over a period of several months, a unique data set was obtained.

Despite the availability of excellent data, the conclusions turned out to be ambiguous. In essence, the data were found to be consistent with two mutually exclusive interpretations: Growth of the response amplitude in proportion to (1) sound intensity and (2) sound pressure diminished by a certain threshold sound pressure. The latter alternative explains the data for a wider range of levels, and this is why a preliminary analysis, published in a conference proceedings volume (Lütkenhöner et al. 2007), shows response amplitude as a function of sound pressure. But in contradiction to signal detection theory (Swets 1961), this interpretation implies the existence of a threshold. To get things straight, an independent modeling study was performed (Lütkenhöner 2008). Starting from wellfounded assumptions about the rate-intensity functions of single auditory nerve fibers (Yates 1990; Yates et al. 1990; Eatock et al. 1991) and the pattern of cochlear excitation caused by a tone (e.g., Russell and Nilsen 1997), formulas for the intensity dependence of the auditory nerve response to a tone were derived. According to these formulas, the response amplitude is, in the low-intensity limit, proportional to intensity. Moreover, the formulas make specific predictions as to how this proportional growth turns into a logarithmic growth, corresponding to a linear increase of the response amplitude with increasing sound level. The present article focuses on the question as to what extent our experimental data are consistent with these theoretical predictions.

\section{MATERIALS AND METHODS}

\section{Subjects}

For the reasons explained in the "Introduction" section, this study was basically confined to collecting high-quality data from the first author (subject S1). Limited measurements in two additional subjects were done with the objective of a rough consistency check. Subject S2 was the second author, and subject S3 was a 20 -year-old student who was paid for her time. All subjects had normal hearing thresholds. The experimental procedures were approved by the Ethics Commission of the Medical Faculty of the University of Münster. Written informed consent was obtained from subject S3 after having explained the nature and purpose of the investigation.

\section{Stimulation}

The stimulus was a Gaussian-shaped tone pulse with a $4-\mathrm{kHz}$ carrier frequency and a full width at half maximum of $0.5 \mathrm{~ms}$. It was transmitted by a short plastic tubing (length of $25 \mathrm{~cm}$ ) from an electrically shielded soundsource (Eartone 3A insert earphone) to an earplug in the subject's right ear. The stimulus level was adjusted relative to a subjective threshold that was determined immediately before the electrophysiological measurements.

\section{Measuring room}

The experiments were carried out in an electrically shielded double-walled anechoic room that extends over all three floors of the building. The inner room (between the tips of the wedges, having a length of $0.9 \mathrm{~m}$ ) has an area of $6.3 \times 7.6 \mathrm{~m}^{2}$ and a clear height of $4.9 \mathrm{~m}$. A listener experiences the feeling of absolute silence in this room, except for self-generated noise such as breathing, rustling of clothes, or stomach rumbling. Consistent with this subjective assessment, the ambient noise in the room is in the noise floor of a Brüel \& Kjaer type 4145 condenser microphone (corresponding to about $10 \mathrm{~dB}(\mathrm{~A})$ ). To get a rough idea as to what extent ambient noise is attenuated in the anechoic room, we tested the presumed weakest point of the acoustic shielding, the passageway between the anechoic room and the adjacent experimenter's room. A tone (about $80-\mathrm{dB}$ sound pressure level [SPL]) was generated by a loudspeaker in the 
middle of the experimenter's room, and the sound level near the first door of the passageway $(2.5 \mathrm{~m}$ from the speaker) was compared with the sound level near the measuring chair in the anechoic room. Although the data were averaged over a period of $500 \mathrm{~s}$, a spectral peak at the stimulus frequency was found only when the first door (the massive external door of the anechoic room) was open. For the frequency range 250$4,000 \mathrm{~Hz}$, we got attenuation factors between 67 and $78 \mathrm{~dB}$. Thus, when all doors are closed, an attenuation factor of the order of $100 \mathrm{~dB}$ or better may be expected. This rough estimate is consistent with attenuation measures published by Grützmacher (1963) for a comparable room.

\section{Determination of the auditory threshold}

The threshold was determined using a two-interval two-alternative forced-choice procedure that was combined with a transformed up-down method converging to $p=0.794$ (Levitt 1971). In the standard measurements, this adaptive procedure changed the level in steps of $1 \mathrm{~dB}$, starting a few decibels above the expected threshold. Figure 1A illustrates the course of events in a single trial. A weak Gaussian-shaped tone pulse of $500 \mathrm{~Hz}$ (full width at half maximum $=8 \mathrm{~ms}$ ) served as an alarm signal. The subjects were instructed to avoid any movement after the alarm, to breathe shallowly (or to hold their breath) until the end of the trial, and to concentrate on the test. The beginning of the first test interval, $500 \mathrm{~ms}$ after the alarm, was indicated by a weak tone pulse of $250 \mathrm{~Hz}$; in all other respects, this signal corresponded to the alarm signal. Identical tone pulses indicated the beginning and the end of the second test interval, at 4 and $8 \mathrm{~s}$, respectively. The test signal was presented five times at intervals of $500 \mathrm{~ms}$; this series was presented exactly in the middle of either interval (the second one in the example shown in Fig. 1A). After the end of each trial, the subjects had to respond by pressing a button. By briefly pressing once or twice they indicated in

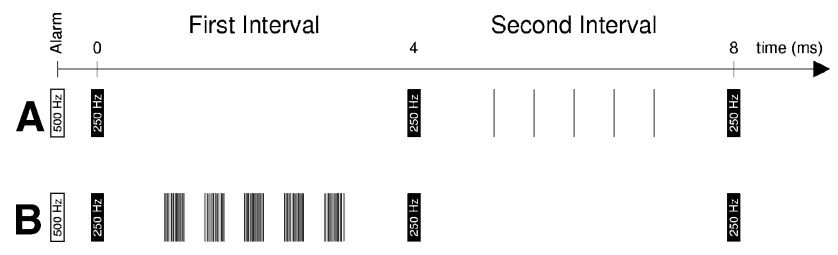

FIG. 1. A Sequence of events during a single trial of the adaptive threshold estimation procedure. After an alarm signal $(500-\mathrm{Hz}$ tone pulse), five repetitions of the test signal are presented in one of two test intervals (marked by $250-\mathrm{Hz}$ tone pulses). In this example, the test signal is a single pulse and the series of test signals is presented in the second test interval. B In supplementary threshold estimations, the single pulse was replaced by a series of two or 16 pulses presented at 16 -ms intervals. The figure illustrates the latter case (series of test signals presented in the first test interval). which interval they most likely perceived the test signal. They did not receive feedback regarding this decision. To show absolute certainty about the response, the subjects had the additional option to prepend a long button press. This forced the control algorithm to immediately continue with a lower level. This option was used especially in the beginning of the procedure to quickly approach a rough threshold. If the subjects had absolutely no idea how to decide, they could leave the decision to the control algorithm by shortly pressing the button thrice. But this option was rarely used.

Three different test signals were considered in this study: A single presentation of our $4-\mathrm{kHz}$ tone pulse and two or 16 presentations in series at intervals of $16 \mathrm{~ms}$ (as in the electrophysiological measurements). Figure 1B illustrates the time course of a single trial for a series consisting of 16 pulses (the five repetitions of the test signal are found in the first test interval now). This test condition may be assumed to be relatively close to the situation during the electrophysiological measurements (for test signals consisting of 32 pulses, the silent periods between the five repetitions of the test signal would disappear, and for a duration of $2.5 \mathrm{~s}$, we would have exactly the same situation as during the electrophysiological measurements). But the levels reported in this article refer to a different threshold: the threshold that was determined for a single pulse as the test signal, i.e., a pulse series of length 1 . This level is defined as $0 \mathrm{~dB} \mathrm{SL}_{1}$ (sensation level for a single pulse as the test signal). In absolute terms, the threshold also depends, of course, on aspects of the testing procedure. Most notably, significantly higher thresholds are obtained if the test signal is presented once rather than five times at 500-ms intervals. A systematic investigation of this point in subject S1 yielded a difference of $3.3 \mathrm{~dB}$ (single pulse as the test signal; mean of eight sessions).

In addition to the routine threshold estimations preceding the electrophysiological measurements (with a single pulse as the test signal), the thresholds for pulse series of length 1,2 , and 16 were compared in supplementary psychoacoustic experiments. The three test signals were presented at random, and the adaptive threshold estimation procedures were independent, except that the control algorithm synchronized the number of reversals between the three processes (a process leading with respect to the number of reversals was stopped until the other processes had drawn even). Thus, the three thresholds were determined quasisimultaneously. The levels were changed in steps of $0.5 \mathrm{~dB}$.

\section{Electrophysiological measurements}

While presenting the tone pulses at 16-ms intervals, the ABR was recorded between vertex and right and 
left earlobe, respectively, using $\mathrm{Ag}-\mathrm{AgCl}$ electrodes. An additional ground electrode was attached to the forehead. The two channels will be referred to as the ipsilateral recording (same side as the stimulation) and the contralateral recording. The recorded potential was amplified by $70 \mathrm{~dB}$, filtered between $0.1 \mathrm{~Hz}$ (first-order RC filter) and $3,000 \mathrm{~Hz}$ (eighth-order Bessel filter), and sampled at a rate of 10,000/s using a SUN workstation equipped with an Analyx ADDA1418 SBus board (for further technical details, see Ross et al. 1999). An epoch, comprising a time window of $14 \mathrm{~ms}$ that started $1 \mathrm{~ms}$ before the stimulus, was considered contaminated by artifacts if the difference between largest and smallest sample differed by more than $63 \mu \mathrm{V}$. Sets of 16 valid epochs were averaged, and the resulting subaverages were stored on disk.

The subjects were sitting reclined on a comfortable chair while reading something of their own choice. Writing notes was also allowed so that the subjects could use the measuring time for limited desk work. While they were aware of the fact that movements should be avoided as far as possible, they also knew that periods with higher muscle activity would represent no principle problem because the affected epochs would be efficiently eliminated by artifactrejection software. The measuring time per session was typically about $2 \mathrm{~h}$, but depended to some extent on the subject's time schedule. The sound level was generally held constant for $15 \mathrm{~min}$. Extreme level differences in subsequent recordings were avoided, but apart from that, the levels were randomized.

In subject $\mathrm{S} 1$, the level range between -3 and $11 \mathrm{~dB}$ $\mathrm{SL}_{1}$ was studied in steps of $2 \mathrm{~dB}$; more than 1,000,000 epochs were averaged for each level. About half of the number of epochs was averaged for 15 and $20 \mathrm{~dB}$. In subject $\mathrm{S} 2$, only the levels 3,7 , and $11 \mathrm{~dB} \mathrm{SL}_{1}$ were considered (about 240,000 averages for each level). The same levels and $1 \mathrm{~dB} \mathrm{SL}_{1}$ in addition were considered in subject S3 (between 200,000 and 430,000 averages per condition). Moreover, also some higher levels (up to $65 \mathrm{~dB} \mathrm{SL}_{1}$ ) were tested in this subject.

\section{Data processing}

After having completed all experiments, the recorded data (subaverages of 16 epochs) were filtered. Moreover, the baseline was adjusted so that the mean potential (averaged over all time points of a subaverage) was 0 . The filter consisted of two components: a low-pass filter that eliminated high-frequency noise and a notch filter that removed the strong steady-state response caused by the repetitive stimulation at $16-\mathrm{ms}$ intervals (corresponding to $62.5 \mathrm{~Hz}$ ). The low-pass filter was an eighth-order Butterworth with a cutoff frequency of $1,000 \mathrm{~Hz}$; the signal was passed through the filter in both forward and reverse directions
(Matlab routine FILTFILT). The effect of the notch filter is illustrated in Figure 2. The response shown as a black curve in the upper panel is dominated by the steady-state component. This component was estimated in the time domain by means of least-squares fit (sine wave displayed as a gray curve). By subtracting this wave from the unfiltered signal, which is equivalent to notch filtering, the curve in the bottom panel was obtained. Faster response components can be recognized much more easily now.

The preprocessed data were averaged over all sessions. Moreover, the standard error of the mean (SEM) was calculated for each time point. The ratio of the mean and SEM corresponds to the $z$ value for a hypothesized mean of 0 (strictly speaking, it is the $t$ value, but for the data considered in this article, the number of degrees of freedom is so high that the $t$ distribution is nearly identical with the standard normal $(z)$ distribution). The $z$ value was used to assess whether an observed peak significantly differed from noise.

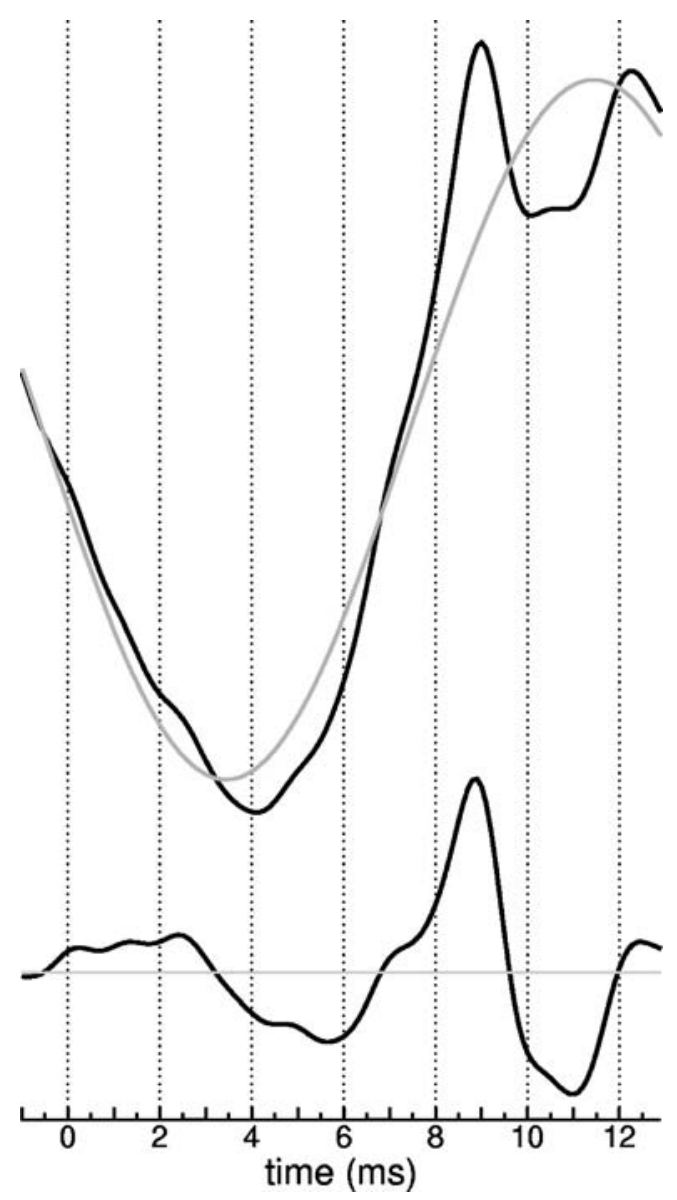

FIG. 2. Notch filtering. The steady-state response related to the repetitive stimulation at 16-ms intervals (black curve in the upper panel) is approximated by a sine wave (gray curve). Subtraction of this wave resulted in the notch-filtered response shown in the bottom panel. 


\section{Level, intensity, sound pressure}

Sound level is specified in $\mathrm{dB}^{\mathrm{S}} \mathrm{SL}_{1}$ throughout this article, i.e., it is defined on a scale that refers to the subjective threshold of a test person (see above). The peak-to-peak equivalent sound pressure level (ppe SPL) corresponding to $0 \mathrm{~dB} \mathrm{SL}_{1}$ is $28 \mathrm{~dB}$ in subject $\mathrm{S} 1$, $26 \mathrm{~dB}$ in subject $\mathrm{S} 2$, and $25 \mathrm{~dB}$ in subject $\mathrm{S} 3$.

In part of the data analysis, sound pressure and intensity are considered rather than sound level. Since 0 dB SPL corresponds to a root-mean-square (RMS) sound pressure of $20 \mu \mathrm{Pa}$, the sound pressure corresponding to a specified $\mathrm{dB} \mathrm{SL}_{1}$ value can be easily calculated. For plane waves, sound pressure $p$ and intensity $I$ are related by the equation $I=p^{2} /(\rho c)$ where $\rho c$ (the product of density $\rho$ and sound speed $c$ ) represents the specific impedance of air (see, e.g., Hartmann 1998). The intensity corresponding to a sound pressure of $20 \mu \mathrm{Pa}$ amounts to approximately $10^{-12} \mathrm{~W} / \mathrm{m}^{2}$ (the exact value depends on atmospheric pressure and temperature). Thus, our $\mathrm{dB}^{\mathrm{S}} \mathrm{SL}_{1}$ values can be converted into approximate intensities as well. However, the considerations presented in this article do not require such conversions; it is completely sufficient to deal with relative sound pressures and relative intensities. For the sake of simplicity, we assume that the reference intensity $I_{\text {ref }}$ and the reference sound pressure $p_{\text {ref }}$ are defined such that the relative intensity $I / I_{\text {ref }}$ and the relative sound pressure $p / p_{\text {ref }}$ are related by the equation

$$
I / I_{\text {ref }}=\left(p / p_{\text {ref }}\right)^{2} .
$$

Modeling the intensity dependence of the response amplitude

As already mentioned in the "Introduction" section, specific predictions about the intensity dependence of the auditory nerve response to a tone were made in a separate modeling study (Lütkenhöner 2008). The most basic results will be briefly summarized now. The equations below are equivalent to their counterparts in the theoretical paper, although their appearance differs to some extent, owing to an altered definition of the model parameters.

Only the simplest model of the theoretical study will be considered here. According to that model, response amplitude $A$ and intensity $I$ are related by the equation

$$
A=\alpha \ln \left(1+I / I_{r e f}\right)
$$

where $\alpha$ is an amplitude factor and $I_{\text {ref }}$ is a reference intensity (as in the previous subsection). In the lowintensity limit, this function reduces to

$$
A=\alpha I / I_{\text {ref }}
$$

which means that response amplitude and intensity are proportional. The high-intensity approximation of this equation can be written as

$$
A=\alpha \ln (10) / 10 \times\left(L-L_{r e f}\right)
$$

where

$$
L=10 \log _{10}(I)
$$

is the sound level corresponding to the intensity $I . L_{\mathrm{ref}}$ is, accordingly, the sound level corresponding to the intensity $I_{\text {ref. }}$. Thus, the model suggests that the response amplitude is, in the high-intensity limit, a linear function of sound level.

To fit the experimental data to Eq. 2, a leastsquares minimization procedure was used. Finding the optimal $\alpha$ for a given reference intensity $I_{\text {ref }}$ merely requires the solution of a linear equation. The remaining problem, the optimization of the parameter $I_{\text {ref }}$, was solved using Brent's method (e.g., Press et al. 1992).

\section{RESULTS}

\section{ABR waveforms}

Figure 3 shows the ABR of subject S3 for sound levels between 65 and $1 \mathrm{~dB} \mathrm{SL}_{1}$. The ipsilateral recording is represented by a black curve, while the contralateral recording is represented by a gray curve. Wave V (in the nomenclature of Jewett and Williston 1971) is marked by an arrow. The wave can be traced over the whole intensity range, whereas other waves are not very distinct at lower levels. Thus, only wave $\mathrm{V}$ is considered in the following analysis, which is confined to levels up to $20 \mathrm{~dB} \mathrm{SL} \mathrm{S}_{1}$.

Figure 4 shows the ABR of subject S1 for levels between 20 and $-3 \mathrm{~dB} \mathrm{SL}_{1}$. Wave $\mathrm{V}$ (in the ipsilateral recording again marked by an arrow) is easily discernible down to $-1 \mathrm{~dB} \mathrm{SL} L_{1}$. At $-3 \mathrm{~dB} \mathrm{SL}_{1}$, at least the downward slope of the wave seems to be clearly distinct from noise. Consistent curves were obtained in subject S2, but only three intensities were tested (Fig. 5).

\section{Amplitude of $A B R$ wave $V$}

Figure 6 shows, separately for each subject, the amplitude of ABR wave $\mathrm{V}$ as a function of level. The ipsilateral recording is represented by black symbols, while the contralateral recording is represented by gray symbols. Error bars indicate twice the SEM. A black solid curve represents the function specified in Eq. 2. The two parameters of this function, amplitude factor $\alpha$ and reference intensity $I_{\text {ref }}$ (or, equivalently, reference level $L_{\text {ref }}$ ), were optimized using a leastsquares fit for the level range 3 to $11 \mathrm{~dB} \mathrm{SL}_{1}$ (results 


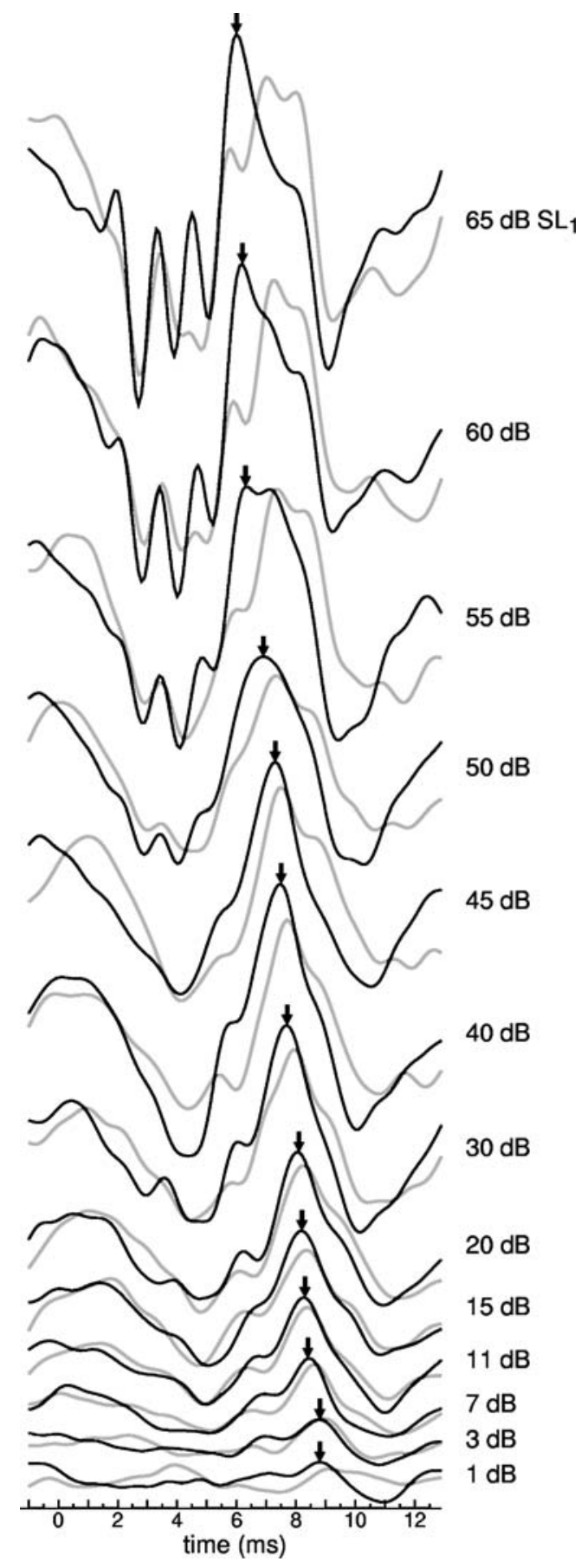

FIG. 3. $A B R$ of subject $S 3$ for levels between 65 and $1 \mathrm{~dB} \mathrm{SL}_{1}$.

provided in Table 1). By choosing this range, all three subjects could be treated in a consistent way. Accounting for higher levels would have been questionable anyway because the model underlying Eq. 2 was developed for very low levels. In subjects S1 and S3, the response amplitudes observed at the two highest intensities are indeed smaller than predicted by the model, which is presumably a consequence of additional compressive components. Most remarkable is that, in subject S1, Eq. 2 makes reasonable predictions regarding the three lowest levels. The high-level approximation of Eq. 2 is represented by a dotted line. In contrast to the exact function, this approximation does not provide a satisfactory description of the data. But it helps to understand the meaning of the reference level $L_{\text {ref: }}$ The high-level approximation becomes 0 at this level.

The dash-dotted lines in Figure 6 represent an unconstrained linear fit to the data (again considering the level range 3 to $11 \mathrm{~dB} \mathrm{SL}_{1}$ ). The data of all three subjects are roughly consistent with the view that, at levels near the subjective threshold, there is a roughly linear relationship between response ampli-

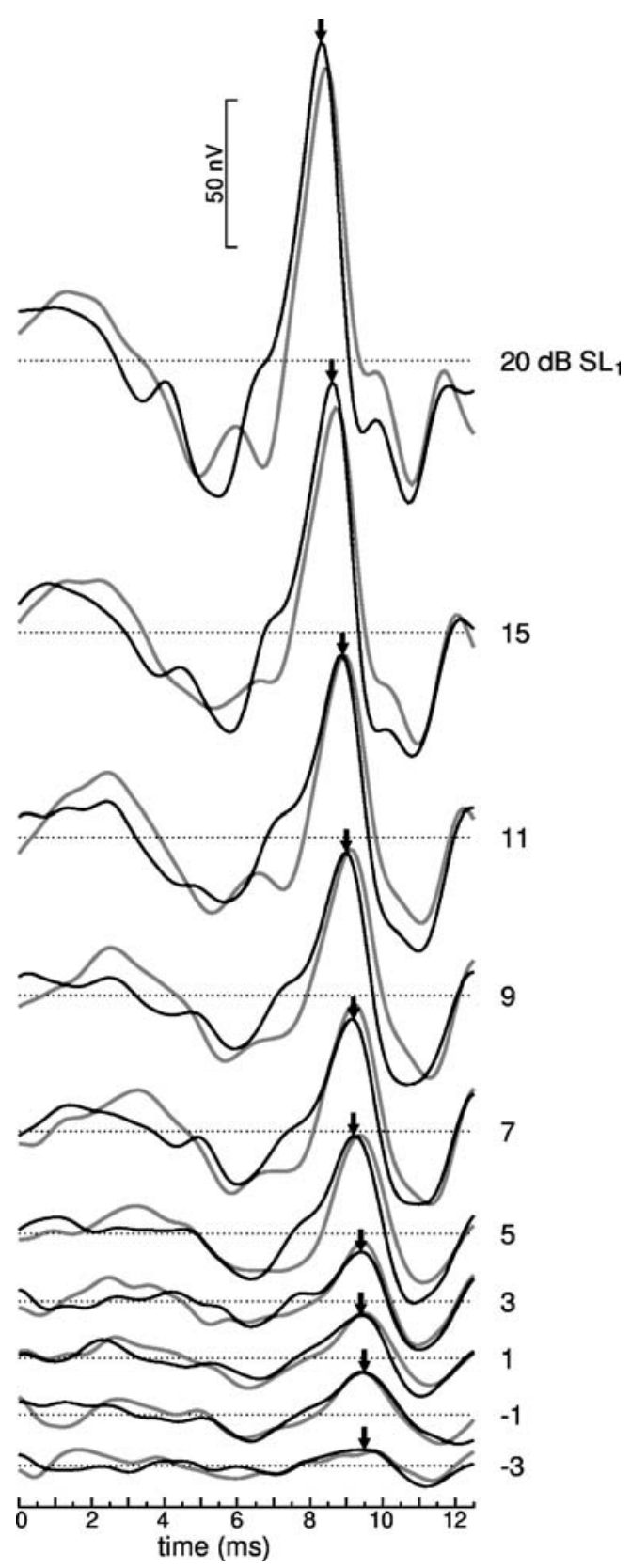

FIG. 4. $A B R$ of subject $S 1$ for levels between 20 and $-3 d_{B} \mathrm{SL}_{1}$. 


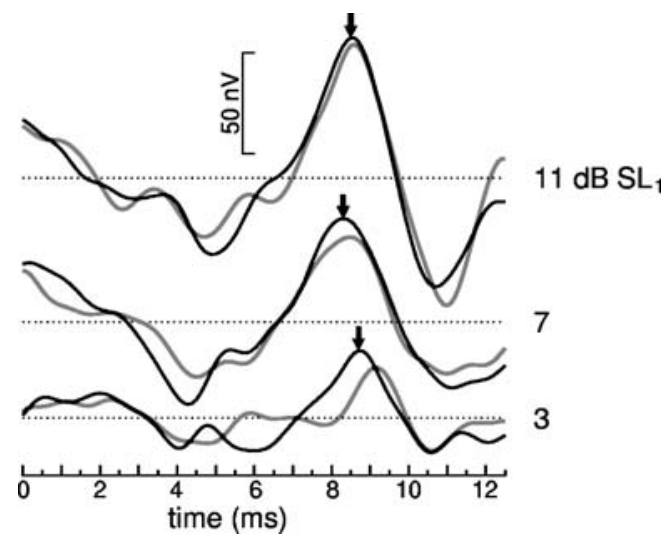

FIG. 5. $A B R$ of subject $S 2$ for levels between 11 and $3 \mathrm{~dB} \mathrm{SL}_{1}$.

tude and level. Clear deviations are only found at extremely low levels (subject S1) and at higher levels (subject S3). The parameter $L_{\text {linear }}$ in Table 1 is the "threshold" that is obtained by extrapolating the linear fit. For the three subjects tested, this "threshold" is a few decibels lower than $L_{\text {ref. }}$. Most instructive are the results for subject $\mathrm{S} 1$. The linear fit describes the data reasonably well between 1 and $20 \mathrm{~dB} \mathrm{SL}_{1}$, but the data clearly disprove the prediction of a threshold at $-0.97 \mathrm{~dB} \mathrm{SL}$.

\section{Statistical testing}

Since the polarity of wave $\mathrm{V}$ is known in advance, the significance of an observed peak can be assessed using a one-sided statistical test. The critical $z$ value corresponding to $p=0.0001$ is 3.72. Except for the lowest level tested, the estimated wave $\mathrm{V}$ amplitudes have $z$ values greater than that critical value. The critical value of 1.65 , corresponding to $p=0.05$, was also exceeded at the lowest level in all subjects (see Table 2).

\section{Supplementary psychoacoustic experiments}

In the ABR recordings in subject S1, the two lowest levels were below the subjective threshold determined at the beginning of a session (see the definition of the $\mathrm{dB} \mathrm{SL}_{1}$ scale in the "Materials and methods" section). Nevertheless, there was always a weak perception of sound, at least when concentrating on the stimulation. The point is that the stimuli were presented in a continuous series at $16-\mathrm{ms}$ intervals. So the question arises to what extent the threshold depends on the

FIG. 6. Level dependence of the amplitude of $A B R$ wave $V$ for subjects S1 (top), S2 (middle), and S3 (bottom). Black symbols ipsilateral recording, gray symbols contralateral recording. Error bars indicate twice the SEM. The solid curve was obtained by fitting the data to Eq. 2, the dotted line is the high-intensity approximation (Eq. 4), and the dash-dotted line represents a linear fit to the data. number of stimuli presented in rapid succession. Table 3 shows the results for two and 16 stimuli; the threshold for a single stimulus served as the reference
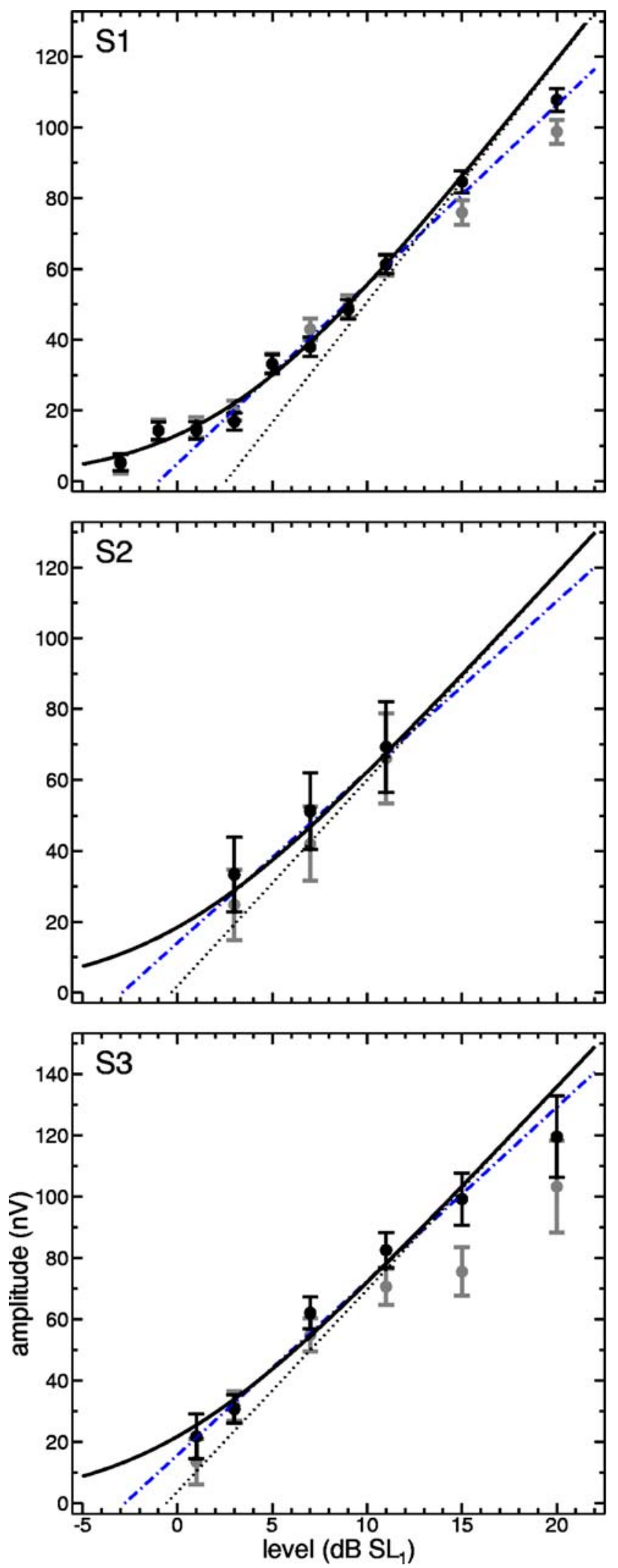
TABLE 1

ABR threshold determined in two different ways

\begin{tabular}{lcrcc}
\hline Subject & $\alpha(n V)$ & $L_{\text {ref }}(d B)$ & $L_{\text {linear }}(d B)$ & $L_{\text {ref }}-L_{\text {linear }}(d B)$ \\
\hline S1 & 29.6 & 2.56 & -0.97 & 3.53 \\
S2 & 25.2 & -0.32 & -2.91 & 2.59 \\
S3 & 28.6 & -0.58 & -2.78 & 2.20 \\
\hline
\end{tabular}

$\alpha$ is the amplitude factor in Eq. 2. $L_{\text {ref }}$ is the sound level that corresponds to the reference intensity $I_{\text {ref }}$. $L_{\text {linear }}$ refers to the level at which a linear approximation of response amplitude versus sound level intersects the abscissa

(0 $\mathrm{dB} \mathrm{SL}_{1}$, by definition). For a pair of stimuli, the mean threshold was $-1.6 \mathrm{~dB} \mathrm{SL}_{1}$, for a series of 16 stimuli the mean threshold was $-5.6 \mathrm{~dB} \mathrm{SL}_{1}$.

\section{DISCUSSION}

\section{ABR amplitudes compared to model predictions}

The main objective of this study was to characterize the level dependence of ABR wave $\mathrm{V}$ near its detection limit. Specific predictions regarding this dependence were made in a companion modeling study (Lütkenhöner 2008). Our analysis consequently focused on the question as to what extent our experimental data are consistent with these predictions. We could show for all three subjects that up to a level of at least $11 \mathrm{~dB} \mathrm{SL}_{1}$ the data agree well with the intensity dependence specified in Eq. 2. We, therefore, conclude that the underlying model, although based on assumptions about the gross response of the auditory nerve, makes valid predictions regarding $\mathrm{ABR}$ wave $\mathrm{V}$.

Three sound level ranges are distinguished in the theoretical paper (Lütkenhöner 2008). The model predicts a more or less proportional relationship between sound intensity and response amplitude for extremely low sound levels (range I) and an approximately linear level dependence of the response amplitude for higher levels (range III). For levels in between (around the reference level $L_{\text {ref }}$ defined in the context of Eq. 5), the model predicts a smooth transition between the two types of growth (range II). Figure 7 suggests that the low-level data of the present

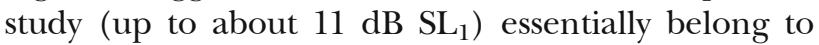

\section{TABLE 2}

Significance analysis for the lowest level tested

\begin{tabular}{lclcc}
\hline Subject & Level $(d B)$ & Recording side & z value & $p$ value \\
\hline S1 & -3 & Ipsilateral & 2.25 & $0.0122^{*}$ \\
& & Contralateral & 1.73 & $0.0418^{*}$ \\
S2 & 3 & Ipsilateral & 3.14 & $0.0008^{* * *}$ \\
& \multirow{2}{*}{ S3 } & Contralateral & 2.49 & $0.0064^{* *}$ \\
& 1 & Ipsilateral & 3.01 & $0.0013^{* *}$ \\
& & Contralateral & 1.85 & $0.0322^{*}$ \\
\hline
\end{tabular}

${ }^{*} p<0.05,{ }^{* *} p<0.01,{ }^{* * *} p<0.001$
TABLE 3

Thresholds for two and 16 stimuli, respectively, presented at 16 -ms intervals

\begin{tabular}{lcccc}
\hline & & \multicolumn{2}{c}{ Estimated thresholds $\left(\mathrm{dB} S L_{1}\right)$} \\
\cline { 4 - 5 } Subject & Sessions & Reversals & 2 stimuli & 16 stimuli \\
\hline S1 & 6 & 48 & -1.6 & -4.9 \\
S2 & 3 & 24 & -1.4 & -5.2 \\
S3 & 3 & 24 & -1.8 & -6.6 \\
Mean & & & -1.6 & -5.6 \\
\hline
\end{tabular}

The threshold for a single stimulus served as the reference (defined as $0 \mathrm{~dB}$ $\mathrm{SL}_{1}$ ). The thresholds of the individual subjects represent the medians of all reversals

the latter range. The figure shows the same data as the upper plot in Figure 6 (subject S1), but level was converted into normalized intensity. To be consistent with the theoretical paper, the normalization was chosen so that the reference level $L_{\text {ref }}$ (see Table 1) corresponds to a normalized intensity of 1 . As in Figure 6, the exact model (Eq. 2) is represented by a solid curve, whereas the high-intensity approximation (Eq. 4) is represented by a dotted curve. Besides that, this figure also shows the low-intensity approximation (Eq. 3) as a dashed line. Except for the lowest and highest levels, the data are clearly located between the dashed line and dotted curve. This suggests that we observed a transition from a linear to a logarithmic growth of the response amplitude.

The empirical linear fit shown as a dash-dotted line in Figure 6 basically corresponds to the amplitude growth predicted for range III of the theoretical paper. The fact that this fit explains the data as well (except for the lowest levels considered in subject S1) is not at all contradictory to the above conclusion that the low-level data of the present study basically belong to intensity range II. The point is that it is not permissible to interpret the result of such an unconstrained linear fit as the high-intensity approximation of the model. This would lead to the contradiction that the data are explained by an approximation of the model, but not by the full model itself.

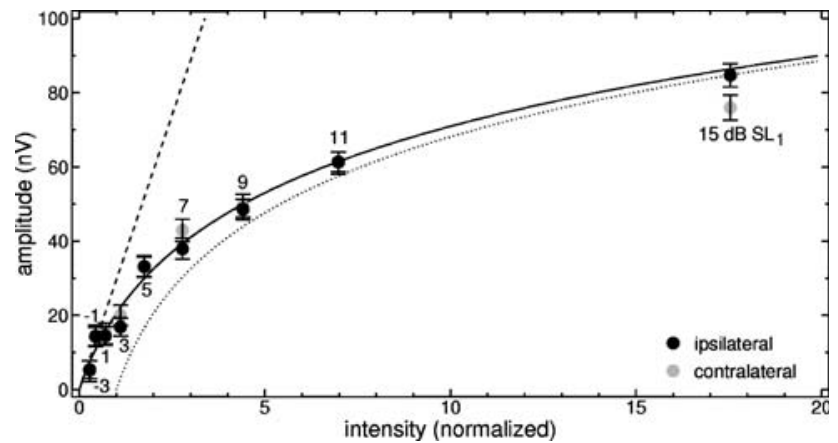

FIG. 7. Wave $V$ amplitude as a function of normalized intensity (same data as in the upper plot of Fig. 6). 
An alternative interpretation of the data: linear dependence on sound pressure near threshold

The fact that our data are in good agreement with the companion modeling study (Lütkenhöner 2008) does not imply that they conclusively disprove alternative interpretations. Based on the data alone it is, in particular, impossible to reject the hypothesis that the response amplitude near the detection limit is linearly related to sound pressure. Figure 8 shows the same data as Figure 7 and the upper plot of Figure 6, but now the abscissa represents normalized sound pressure, defined as the square root of normalized intensity. The solid curve represents the model (Eq. 2), as in the previous figures. Up to a level of about $9 \mathrm{~dB}$ $\mathrm{SL}_{1}$, this curve basically coincides with the dash-dotted line, which represents a linear fit to the data in the range -3 to $9 \mathrm{~dB} \mathrm{SL}_{1}$. We may conclude that the data are not only consistent with the model, but also with the alternative interpretation that the response amplitude near the detection limit is a linear function of sound pressure.

This ambiguity in the data has been predicted in the companion theoretical study. In principle, the issue could be resolved by a single additional measurement. When plotted as a function of sound pressure, the dash-dotted line in Figure 8 suggests the existence of a threshold at a normalized sound pressure of 0.266 , corresponding to $-8.9 \mathrm{~dB} \mathrm{SL}_{1}$, i.e., almost $6 \mathrm{~dB}$ below the lowest level in our experiments. Measuring a response at that level would clearly disprove the sound pressure hypothesis. However, such an experiment is virtually impossible because the measuring time would exceed the time required for the present study by an order of magnitude, as the following estimation shows. If we assume that response amplitude and intensity are proportional in the low-intensity limit, lowering the sound level by $6 \mathrm{~dB}$ would reduce the response amplitude by a factor of 4; thus, maintaining the signal-to-noise ratio would require a 16 -fold increase of the number of averages.

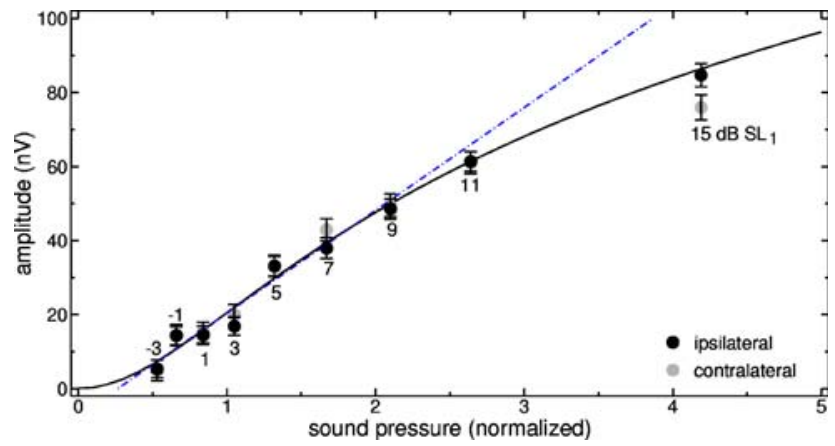

FIG. 8. Wave $\mathrm{V}$ amplitude as a function of normalized sound pressure (same data as in the upper plot of Fig. 6).
The above consideration suggests that noninvasive ABR recordings in humans may not be sensitive enough to prove the correctness of the model for extremely low levels. But this should not be considered a problem because two aspects are to be distinguished when discussing the merits of the model. The first question is whether the model makes correct predictions for the sound level range accessible to noninvasive ABR experiments. The answer is that our low-level data do not show any contradiction to the model. The second question is whether the model allows, in an experiment of thought, a continuation to lower levels that are accessible only to invasive studies. The answer follows from the construction of the model: At ultra low levels, the model reflects nothing else than the rate-intensity functions of the most sensitive auditory nerve fibers.

\section{Definition of an ABR threshold}

The model denies the existence of a threshold, which means that a loss in signal-to-noise ratio due to a reduction in sound level can always be compensated for by increasing the measuring time, at least in theory. Our data are consistent with this view. Although single sessions did not allow estimating reliable responses at the lowest levels, clear responses were obtained by averaging over all sessions.

Notwithstanding the fact that an absolute threshold does not exist, an ABR that significantly rises above the noise floor is found only when the sound level exceeds a certain limit, and this minimum level is usually considered the ABR threshold (e.g., Sininger 2007). The problem is that a threshold defined that way depends on methodological issues such as measuring time. At least in theory, this problem may be overcome by defining a threshold based on Eq. 2. The function specified in this equation is determined by just two parameters so that measurements at two different sound levels (not necessarily including an extremely low level, as demonstrated in Fig. 6 for subject S1) are, in principle, sufficient to estimate them. Although the function may be considered an explicit mathematical formulation of the idea that an absolute threshold does not exist, it offers a natural definition of a de facto threshold. The point is that the reference intensity $I_{\text {ref }}$ determines the transition from a linear to a logarithmic growth of the response amplitude, and only the latter is observable in a typical experiment.

The reference levels determined for our three subjects are compiled in Table 1. Speculations about interindividual differences are not reasonable considering the low number of subjects. Thus, we simply conclude that the ABR threshold, defined as the reference level, is roughly $0 \mathrm{~dB} \mathrm{SL}_{1}$. It must be 
emphasized at this point that a perfect match between ABR threshold and subjective threshold is not to be expected because both thresholds depend on relatively arbitrary definitions and the underlying experiments differ in several respects.

\section{Temporal integration}

The $\mathrm{dB} \mathrm{SL}_{1}$ scale refers to the perception of five stimulus presentations at 512-ms intervals (see the "Materials and methods" section), whereas, in the electrophysiological experiments, the stimuli were continuously presented at 16 -ms intervals. The relatively high stimulus repetition rate explains why the two lowest levels tested in subject $\mathrm{S} 1 \quad(-3$ and $-1 \mathrm{~dB}$ $\mathrm{SL}_{1}$ ) resulted in a weak perception. This kind of phenomenon is often referred to as temporal integration. Our supplementary psychoacoustic experiments are roughly consistent with the idea that each doubling of the number of stimuli results in a threshold reduction by $1.5 \mathrm{~dB}$, corresponding to a factor of $\sqrt{2}$ with respect to stimulus intensity. This is exactly the expected outcome of a multiple-look YesNo experiment (Swets et al. 1959; Luce 1963; Viemeister and Wakefield 1991).

Assuming that the temporal integrator is located peripheral to the site of spike generation in the auditory nerve, as suggested by Heil and Neubauer (2003), the wave V amplitude near the detection limit should depend on the stimulus repetition rate. Our electrophysiological experiment was not designed to investigate this aspect because the only parameter varied was sound level. At least, our data do not conflict with the idea that the phenomenon of temporal integration has a peripheral origin. Assuming that the temporal integrator is located centrally to the generators of ABR wave $\mathrm{V}$, our results may add an interesting aspect to the ongoing discussion about temporal integration (e.g., Krishna 2006; Meddis 2006). The finding that, at the detection limit, there is basically a linear relationship between sound pressure and wave V amplitude (although-according to our model - this is only an approximation) appears to fit nicely to the idea that the auditory system integrates sound pressure (Heil and Neubauer 2003; Neubauer and Heil 2004). A central integrator would "see" input activity linearly increasing with sound pressure, and temporal integration of this activity would appear as sound pressure integration.

\section{ACKNOWLEDGMENT}

The authors thank Ms. Katrin Zobel for carefully performing the extremely tedious experiments.

\section{REFERENCES}

Dallos P. Response characteristics of mammalian cochlear hair cells. J. Neurosci. 5:1591-1608, 1985.

Eатоск RA, WeIss TF, Отто KL. Dependence of discharge rate on sound pressure level in cochlear nerve fibers of the alligator lizard: implications for cochlear mechanisms. J. Neurophysiol. 65:1580-1597, 1991.

Eggermont JJ, Odenthal DW. Action potentials and summating potentials in the normal human cochlea. Acta Otolaryngol. Suppl. 316:39-61, 1974.

ElBerLing C, Don M. Threshold characteristics of the human auditory brain stem response. J. Acoust. Soc. Am. 81:115-121, 1987.

Grützmacher M. Der neue schalltote Raum der physikalischtechnischen Bundesanstalt. Acustica. 5:373-376, 1963.

Hartmann WM. Signals, Sound, and Sensation. New York, Springer, 1998.

Heil P, Neubauer H. A unifying basis of auditory thresholds based on temporal summation. Proc. Natl. Acad. Sci. U. S. A. 100:61516156, 2003.

Jewett DL, Williston JS. Auditory-evoked far fields averaged from the scalp of humans. Brain. 94:681-696, 1971.

KrISHNA BS. Comment on "Auditory-nerve first-spike latency and auditory absolute threshold: a computer model" [J. Acoust. Soc. Am. 119, 406-417 (2006)]. J. Acoust. Soc. Am. 120:591-593, 2006.

LevirT H. Transformed up-down methods in psychoacoustics. J. Acoust. Soc. Am. 49:467-477, 1971.

Lopez-Poveda EA, Eustaquio-Martin A. A biophysical model of the inner hair cell: the contribution of potassium currents to peripheral auditory compression. J. Assoc. Res. Otolaryngol. 7:218-235, 2006.

LuCE RD. Detection and recognition. In: Luce RD, Bush RR, Galanter E (eds) Handbook of Mathematical Psychology, Vol. I. New York, Wiley, pp. 103-189, 1963.

LÜTKENHÖNER B. Threshold and beyond: modeling the intensity dependence of auditory responses. J. Assoc. Res. Otolaryngol 9:102-121, 2008.

LÜTKENHÖNER B, KLEIN JS. Auditory evoked field at threshold. Hear. Res. 228:188-200, 2007.

LÜtKenHÖNER B, KLeIN JS, SEITHER-PreisLer A. Near-threshold auditory evoked fields and potentials are in line with the Weber-Fechner law. In: Kollmeier B, Klump G, Hohmann V, Langemann U, Mauermann M, Uppenkamp S, Verhey J (eds) Hearing-From Sensory Processing to Perception. Berlin, Springer, pp. 215-225, 2007.

MedDIS R. Reply to comment on "Auditory-nerve first-spike latency and auditory absolute threshold: a computer model". J. Acoust. Soc. Am 120:1192-1193, 2006.

Neubauer H, Heil P. Towards a unifying basis of auditory thresholds: the effects of hearing loss on temporal integration reconsidered. J. Assoc. Res. Otolaryngol 5:436-458, 2004.

Press WH, Teukolsky SA, Vetterling WT, Flannery BP. Numerical recipes in C. The Art of Scientific Computing. Cambridge, Cambridge University Press, 1992.

Robles L, Ruggero MA. Mechanics of the mammalian cochlea. Physiol. Rev. 81:1305-1352, 2001.

Ross B, LütKenhöner B, Pantev C, Hoke M. Frequency-specific threshold determination with the CERAgram method: basic principle and retrospective evaluation of data. Audiol. Neurootol 4:12-27, 1999.

Russell IJ, NiLSEN KE. The location of the cochlear amplifier: spatial representation of a single tone on the guinea pig basilar membrane. Proc. Natl. Acad. Sci. U. S. A 94:2660-2664, 1997.

SININGER YS. The use of auditory brainstem response in screening for hearing loss and audiometric threshold prediction. In: Burkard RF, Eggermont JJ, Don M (eds) Auditory Evoked Potentials. 
Basic Principles and Clinical Applications. Philadelphia, Lippincott Williams \& Wilkins, pp. 254-274, 2007.

SWETS JA. Is there a sensory threshold? Science. 134:168-177, 1961. Swets JA, Shipley EF, McKey MJ, Green DM. Multiple observations of signals in noise. J. Acoust. Soc. Am 31:514-521, 1959.

Versnel H, Schoonhoven R, Prijs VF. Single-fibre and whole-nerve responses to clicks as a function of sound intensity in the guinea pig. Hear. Res. 59:138-156, 1992.
Viemeister NF, Wakefield GH. Temporal integration and multiple looks. J. Acoust. Soc. Am. 90:858-865, 1991.

YATES GK. Basilar membrane nonlinearity and its influence on auditory nerve rate-intensity functions. Hear. Res. 50:145-162, 1990.

YATES GK, Winter IM, RoBertson D. Basilar membrane nonlinearity determines auditory nerve rate-intensity functions and cochlear dynamic range. Hear. Res. 45:203-219, 1990. 